\title{
An optimal network dimensioning and initial energy assignment minimizing the monetary cost of a heterogeneous WSN
}

\author{
Cüneyt Sevgi ${ }^{\# 1}$, Altan Kocyigit ${ }^{* 2}$ \\ \# Department of Computer Technology and Information Systems, Bilkent University \\ Ankara, Turkey \\ ${ }^{1}$ csevgi@bilkent.edu.tr \\ * Department of Information Systems, Middle East Technical University \\ Ankara, Turkey \\ ${ }^{2}$ kocyigit@metu.edu.tr
}

\begin{abstract}
In this paper, a novel method is proposed to dimension a randomly deployed heterogeneous Wireless Sensor Network (WSN) of minimum monetary cost satisfying minimum coverage and minimum lifetime requirements. We consider WSNs consisting of two different types of nodes clusterheads and ordinary sensor nodes, randomly deployed over a sensing field. All devices are assumed to be stationary and have identical sensing capabilities. However, the clusterheads are more energetic and powerful in terms of processing and communication capabilities compared to sensor nodes. For such a network, finding minimum cost WSN problem is not a trivial one, since the distribution of the mixture of two different types of devices and the batteries with different initial energies in each type of device primarily determine the monetary cost of a WSN. Therefore, we formulated an optimization problem to minimize the monetary cost of a WSN for given coverage and lifetime requirements. The proposed optimization problem is solved for a certain scenario and the solution is validated by computer simulations.
\end{abstract}

\section{INTRODUCTION}

Over the last few years, due to the recent promising advances in wireless and microsensor technologies, WSN applications are emerging as a new way to monitor phenomena in hostile, inaccessible, and harsh physical environments. A typical WSN consists of sheer number of small, batteryoperated sensor nodes with wireless communication, moderate processing and storage capabilities. In such a network, it is vital to analyze the coverage, lifetime and cost problem in order to gain benefit from it.

Due to the large variety of possible WSN applications, coverage problem is subject to a choice of definition. We define coverage as the extent of monitoring achieved by the sensors while sensing the phenomenon in a physical space for a certain deployment scenario. When the sensing field is entirely covered, this is called full coverage (i.e.,100\%). If a random deployment method is used, full coverage would require infinitely many sensor nodes to be deployed [1] which makes the WSN application economically infeasible. Typically, an acceptable degree of partial coverage is preferred in randomly deployed WSN applications. Moreover, coverage can be improved by increasing the sensor node density, which definitely increases the monetary cost of the WSN.

Due to the nodes' scarce energy resources, lifetime is of paramount importance for gaining benefit from a WSN application. To prolong lifetime, one could increase the number of nodes deployed and/or equip the nodes with high capacity batteries, which further increase the WSN's monetary cost.

In this paper, we consider a statically clustered, randomly deployed heterogeneous WSN. In this heterogeneous network, there are two types of devices, namely clusterheads and sensor nodes. These nodes typically consume different amounts of energy since their functions are usually different. Due to their different energy consumption behavior, it is important to equip the devices with optimal initial energy such that leftover energy (i.e., wasted energy) in the nodes is minimum at the end of the lifespan of the WSN. On the other hand, equipping the devices with different initial energies implies that each type of device has a different cost. Increasing the initial energy may prolong the WSN lifetime at the expense of more money. Therefore, to have a cost-effective WSN, there is an optimum mixture of different types of devices equipped with optimal batteries that satisfy the coverage and lifetime requirements.

The novelty of this work is that it treats cost, coverage and lifetime problem in randomly deployed WSNs within a unified framework. This work differs from existing cost and lifetime optimization problems in two key ways. First, the concept of acceptable degree of partial coverage is used, which makes the WSN application economically feasible. Second, we provide a generic framework to relate the cost of a WSN with the number of devices deployed and the initial energy levels of devices. This analysis yields key insights for treating cost, coverage and lifetime unlike several existing approaches that address only one or two of these as separate problems.

The remainder of this paper is organized as follows. Section II includes an overview of our proposed model and necessary assumptions. In Section III, we review the coverage and cluster size concepts. Section IV discusses the issues related 
to the monetary cost of a WSN application and presents the formulation for cost optimization. Section V gives the solution of the optimization problem for a sample scenario and computer simulation results to validate the solution. Section VI concludes the paper.

\section{OUR NETWORK MODEL}

The sensor network model employed in this paper is illustrated in Fig.1. We consider a randomly deployed WSN, which consists of two types of nodes: clusterheads and sensors, each of which may have varying communication capabilities, initial energy, and processing power. Both sensors and clusterheads have identical sensing capabilities and their sensing region is assumed to be a perfect disk. The sensing range, $r_{s}$, is defined as the radius of the sensing region. We primarily consider partial coverage and denote the targeted level of coverage as $P_{\text {cov }}$, the probability of a point in sensing field $D$ is sensed by at least one node.

Sensors can only transmit their sensing data to the associated clusterhead, and clusterheads transmit the aggregated data to the sink. The association between sensors and clusterheads is determined with a "nearest reachable clusterhead" approach, and at any given time instant, each sensor is assumed to belong to at most one cluster. A sensor can communicate with a clusterhead if it is within the transmitting range $r_{t s}$ of the sensor node. A clusterhead can communicate with the sink if it is within the transmitting range $r_{t h}$. The devices in this network do not use adaptive power control schemes. We also assume that clusterheads have the ability to send the data "directly" to the sink. Moreover, no replenishment and recharging are anticipated.

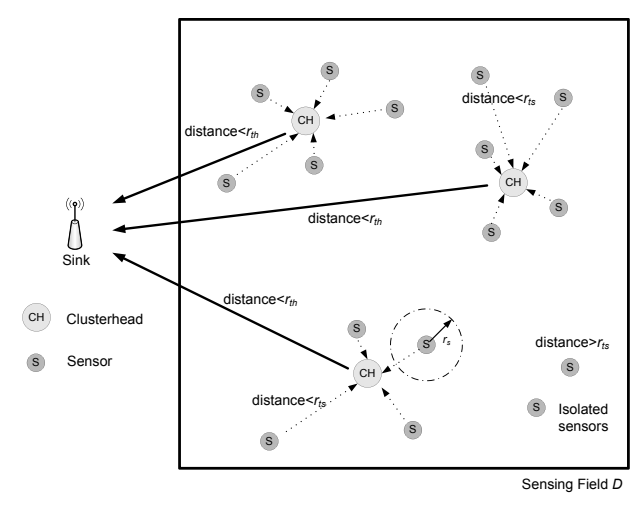

Fig. 1. Cluster hierarchy of the proposed WSN model

Suppose we have a WSN of $N_{S}$ sensors and $N_{H}$ clusterheads deployed randomly over a sensing field $D$ (See Fig.1). Each sensor and clusterhead generate data as they monitor their vicinity. And further suppose that a sensor node can send one packet periodically (i.e.,round) to the associated clusterhead. In the clusterheads, at the end of a round, packets from the cluster members are aggregated into a single relatively longer packet to provide energy efficiency and are sent to the sink. The operation of our model is indeed very similar to that of LEACH (Low-Energy Adaptive Clustering Hierarchy) [2] as it is divided into rounds.

In our model, there are essentially two phases, namely cluster formation and steady state. After deployment, the operation of WSN begins with the cluster formation phase. This phase is performed only once to determine which sensor node will be associated with which clusterhead and to decide on the necessary sensor node transmission schedule to be used in the steady state phase.

In the steady state phase, the sensed data received from the cluster members are forwarded directly to the sink by clusterheads on a regular basis. Every successful operation in the steady state phase is called a "round" (See Fig.2) and is denoted by $R$. In each round, sensor nodes send the sensed data to the clusterhead in the scheduled TDMA (Time Division Multiple Access) slots, and the clusterhead aggregates and sends data to the sink.

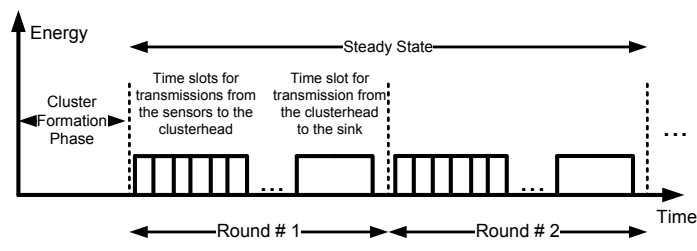

Fig. 2. Cluster Formation and Steady State Phases in a cluster

\section{Coverage And Cluster Size}

Coverage is one of the fundamental issues in WSNs. Cost and lifetime analysis of a WSN application cannot be considered in isolation while ignoring the coverage requirement. The coverage problem in randomly deployed WSNs is different from the coverage problem in deterministic deployment. As full coverage would require infinitely many sensor nodes to be deployed for randomly deployed WSNs, partial coverage is more viable with random deployment. In this paper, we used the connected coverage model proposed in [3]. The area covered by a clusterhead together with the sensors connected to it, is defined as "cluster size", $S_{\text {cluster }}$. By using coverage, connectivity, and $S_{\text {cluster }}$, the coverage $P_{\text {cov }}$ achieved by randomly deployed $N_{H}$ clusterheads and $N_{S}$ sensors over a sensing field of $D$ is found in [3] as :

$$
P_{\text {cov }}=1-e^{-\frac{N_{H} S_{\text {cluster }}}{D}}
$$

where, for $r_{t s} \leq 2 r_{s}$

$S_{\text {cluster }}=\pi\left(r_{s}+r_{t s}\right)^{2}+\frac{2 \pi}{\alpha}\left[\left(\frac{1}{\alpha}-r_{s}\right)\left(1-e^{-\alpha r_{t s}}\right)-r_{t s}\right]$

and for $r_{t s}>2 r_{s}$

$$
\begin{aligned}
S_{\text {cluster }} & =\pi\left(r_{t s}+r_{s}\right)^{2}-\pi r_{t s}\left(r_{t s}-2 r_{s}\right) e^{-2 \alpha r_{s}}- \\
& \frac{2 \pi}{\alpha^{2}}\left[\left(\alpha\left(r_{t s}-r_{s}\right)-1\right)\left(1-e^{-\alpha r_{s}}\right)+2 \alpha r_{s}\right]
\end{aligned}
$$

In the above formulations, $\alpha=n_{s} r_{s} / 2 r_{t s}^{2}$ and $n_{s}$ is the average number of sensors connected to a single clusterhead, 
which can be found as [3]:

$$
n_{s}=\frac{N_{S}}{N_{H}}\left(1-e^{-\frac{N_{H} \pi r_{t s}^{2}}{D}}\right)
$$

\section{Monetary Cost of A WSN}

The monetary cost of a WSN is yet another important performance parameter for a WSN application as it determines whether the application is feasible or not, depending on the cost budget constraint. Typically, a WSN functions for a targeted lifetime at a minimum cost, or operates as long as possible under a certain cost budget. In this paper, we consider the former problem.

By upgrading the battery capacity of devices and by increasing the number of nodes, one can prolong the targeted lifetime. Thus, both of the these methods may lead to an increase of lifetime at the expense of more cost. This lifetime-cost tradeoff is the driver behind this study. As far as our network model is concerned, there are two types of nodes with different functionalities and capabilities. Therefore, the cost associated with clusterheads and that associated with sensors are denoted as $C_{H}$ and $C_{S}$, respectively. Each type of device is composed of a hardware component and a battery providing the power for this hardware. A clusterhead may have superior hardware compared to a sensor node or similarly may have more initial energy than a sensor node or both. The cost of a sensor node, $C_{S}$, is the cost of its hardware unit, $C_{h w}$, added to the cost of its battery $C_{b t y}$. Similarly, the cost of a clusterhead, $C_{H}$, can be represented as the sum of the cost of its hardware unit, $C_{H W}$, and its battery $C_{B T Y}$. Thus, the monetary cost of a WSN $C_{W S N}$ can be found as:

$$
C_{W S N}=N_{H} \cdot C_{H}+N_{S} \cdot C_{S}
$$

The cost differentiation between a clusterhead and a sensor node depends on a wide variety of features of the devices, such as transmitting and sensing ranges, availability of adaptive power control, processing power, storage capacity, and initial energies etc.

In the literature, for some heterogeneous WSNs [4], heterogeneity implies that a set of nodes simply has more initial energy than others while the entire network has identical hardware components (i.e., $C_{h w}=C_{H W}$ ). According to this approach, we can say that each sensor node and clusterhead may have different initial energies. If we use identical battery cells with identical energies, each sensor node and clusterhead will have a different number of these cells. Therefore, it is required to determine the number of cells in each type of device for a given network lifetime $R$. By using these, the monetary cost $C_{W S N}$ of this specific WSN can be rewritten as:

$$
C_{W S N}=N_{H} \cdot\left(C_{H W}+K . C_{\text {cell }}\right)+N_{S} \cdot\left(C_{H W}+k \cdot C_{c e l l}\right)
$$

where $k$ and $K$ are the number of cells used by each sensor and clusterhead respectively and the monetary cost of battery in each type of device is found as the cost a single cell $C_{c e l l}$ multiplied with the number of cells used.

\section{A. Cost Optimization Formulation}

Using the monetary cost of WSN discussed in Eqn.5, the cluster size concept given through Eqn.1 to 4, and energy model and data dissemination technique in [2], we formulated a minimization problem for the cost of a WSN. The formulation is given as:

$$
\begin{aligned}
\min C_{W S N} & =\min N_{H} \cdot C_{H}+N_{S} \cdot C_{S} \\
& \text { subject to } \\
& 1-e^{-\frac{N_{H} S_{\text {cluster }}}{D}} \geq P_{\text {cov }} \\
& \text { K.e } e_{\text {cell }}-\left(E_{c-\text { formation }}+\left(E_{S Y N C}\right.\right. \\
& \left.\left.+E_{\text {s-state }}+E_{A G G}\right) \cdot R\right) \geq 0 \\
& k . e_{\text {cell }}-\left(e_{c-\text { formation }}+\left(E_{S Y N C}\right.\right. \\
& \left.\left.+e_{s-\text { state }}\right) \cdot R\right) \geq 0 \\
& N_{H}, N_{S}, k, K \in \mathbb{Z}^{+}
\end{aligned}
$$

Energy dissipation model is one of the most critical issues in the design and operation of WSNs, due to its direct impact on the WSN lifetime. We essentially adopt the energy dissipation model used in [2]. Table.I lists the necessary notation used in our model.

We primarily consider the wireless communication power consumption and ignore data processing and sensing power consumptions. While computing the energy dissipation for transmissions, we exploited different channel models, which are also pursued in [2].

Our objective is to find the optimum values for $N_{S}, N_{H}$, $K$, and $k$ to minimize cost for given $P_{\text {cov }}, R, r_{s}, r_{t s}, r_{t h}, D$, $e_{\text {cell }}, C_{H W}, C_{\text {cell }}, \rho, \alpha, \beta_{f s}, \beta_{m p}, R_{S Y N C}, R_{A G G}, R_{J O I N}$, $R_{S C H E}, R_{A D V}, R_{D A T A}, E_{A G G}$, and the sink's location.

The set of constraints in Eqn.7 can be interpreted as follows. The first constraint focuses on partial coverage requirement. $P_{\text {cov }}$, which exploits the cluster size concept, is the minimum threshold value for coverage. The second and third constraints, which are related to the initial energy at each clusterhead and sensor, enforce that the energy dissipated for transmissions, receptions, and aggregations should not exceed the initial energy supplies $K . e_{c e l l}$ and $k . e_{c e l l}$ respectively. In these constraints, $E_{c-\text { formation }}, E_{S Y N C}, E_{s-\text { state }}$, and $E_{A G G}$ are energy dissipation for cluster formation, synchronization, steady state, and aggregation operations respectively and have been given in Table.I. Lastly, the fifth constraint imposes $N_{H}, N_{S}, K$ and $k$ to be all positive integers.

Note that, in the above formulation, we tacitly assumed that every cluster has an equal number of sensor nodes, $n_{s}$. Definitely, this will not be the case in random deployment. However, this simplifying assumption provides acceptable approximate solutions as will be demonstrated in Section V.

\section{B. Our Heuristic Solution Method}

Eqn.7 could have been solved by using a conventional Mixed Integer Nonlinear Programming (MINP) solver. However, there are a few constraints and we have a quite simple objective function. Therefore, we performed an heuristic search with respect to system parameters: $P_{\text {cov }}, R, r_{s}, r_{t s}, r_{t h}, D$, $e_{\text {cell }}, C_{H W}, C_{\text {cell }}, \rho, \alpha, \beta_{f s}, \beta_{m p}, R_{S Y N C}, R_{A G G}, R_{J O I N}$, $R_{S C H E}, R_{A D V}, R_{D A T A}, E_{A G G}$, and sink's location. 
TABLE I

SUMMARY OF VARIABLES IN ENERGY DISSIPATION MODEL

\begin{tabular}{|c|c|}
\hline Parameter & Description \\
\hline \multirow[t]{2}{*}{$E_{c-\text { formatio }}$} & $\begin{array}{l}\text { The energy dissipated by a clusterhead (EDC) to } \\
\text { perform the cluster formation phase ( }=E_{A D V}^{t}+ \\
\left.E_{S C H E}^{t}+E_{J O I N-R E Q}^{r}\right)\end{array}$ \\
\hline & $\begin{array}{l}\text { - } E_{A D V}^{t}=\mathrm{EDC} \text { to broadcast } R_{A D V} \text { bits of } \\
\text { advertisement frame }\left(=\alpha+\beta_{f s} \cdot r_{t h}^{2} \cdot R_{A D V}\right) \\
\text { - } E_{S C H E}^{t}=\mathrm{EDC} \text { to broadcast } R_{S C H E} \text { bits } \\
\text { of schedule notification frame }(=\alpha+ \\
\left.\beta_{f s} \cdot r_{t h}^{2} \cdot R_{S C H E}\right) \\
\text { - } E_{J O I N-R E Q}^{r}=\text { EDC to receive } \\
n_{s} \cdot R_{J O I N-R E Q} \text { bits of join-request } \\
\text { frames }\end{array}$ \\
\hline \multirow[t]{2}{*}{$e_{c-\text { formation }}$} & $\begin{array}{l}\text { The energy dissipated by a sensor (EDS) } \\
\text { to perform the cluster formation phase (= } \\
\left.e_{J O I N-R E Q}^{t}+e_{A D V}^{r}+e_{S C H E}^{r}+e_{J O I N-R E Q}^{r}\right)\end{array}$ \\
\hline & $\begin{array}{l}\text { - } e_{J O I N-R E Q}^{t}=\text { EDS to trans- } \\
\text { mit } R_{J O I N-R E Q} \text { bits of join- } \\
\text { request frame to the clusterhead } \\
\left(=\alpha+\beta_{f s} \cdot r_{t s}^{2} \cdot R_{J O I N-R E Q}\right) \\
\text { - } e_{A D V}^{r}=\mathrm{EDS} \text { to receive } R_{A D V} \text { bits of ad- } \\
\text { vertisement frame }\left(=\rho \cdot R_{A D V}\right) \\
\text { - } e_{S C H E}^{r}=\mathrm{EDS} \text { to receive } R_{S C H E} \text { bits of } \\
\text { TDMA schedule frame }\left(=\rho \cdot R_{S C H E}\right) \\
\text { - } e_{J O I N-R E Q}^{r}=\text { EDS to receive }\left(n_{s}-\right. \\
1) \cdot R_{J O I N-R E Q} \text { bits of join-request frame } \\
\left(=\rho \cdot R_{J O I N-R E Q} \cdot\left(n_{s}-1\right)\right)\end{array}$ \\
\hline \multirow[t]{2}{*}{$E_{s-s t a t e}$} & $\begin{array}{l}\text { EDC to perform the steady state phase within a } \\
\text { round }\left(=E_{A G G}^{t}+E_{D A T A}^{r}\right)\end{array}$ \\
\hline & $\begin{array}{l}\text { - } E_{A G G}^{t}=\mathrm{EDC} \text { to transmit } R_{A G G} \text { bits } \\
\text { of aggregated data to the sink }(=\alpha+ \\
\left.\beta_{m p} \cdot r_{t h}^{4} \cdot R_{A G G}\right) \\
\text { - } E_{D A T A}^{r}=\mathrm{EDC} \text { to receive } n_{s} \cdot R_{D A T A} \text { bits } \\
\text { of data frame }\left(=\rho \cdot R_{D A T A} \cdot n_{s}\right)\end{array}$ \\
\hline \multirow[t]{2}{*}{$e_{s-s t a t e}$} & $\begin{array}{l}\text { EDS to perform the steady state phase within a } \\
\text { round }\left(=e_{D A T A}^{t}\right)\end{array}$ \\
\hline & $\begin{array}{l}\text { - } e_{D A T A}^{t}=\text { EDS to transmit } R_{D A T A} \text { bits of } \\
\text { data to the associated clusterhead }(=\alpha+ \\
\left.\beta_{f s} \cdot r_{t s}^{2} \cdot R_{D A T A}\right)\end{array}$ \\
\hline$E_{S Y N C}$ & $\begin{array}{l}\text { EDS or EDC to receive } R_{S Y N C} \text { bits of synchro- } \\
\text { nization stream from the } \operatorname{sink}\left(=\rho .\left(R_{S Y N C}\right)\right)\end{array}$ \\
\hline$E_{A G G}$ & $\begin{array}{l}\text { EDC to aggregate } 1 \text { bit of data from a received } \\
\text { signal }\end{array}$ \\
\hline$\alpha$ & Energy dissipated in the transmitted circuit \\
\hline$\beta_{f s}$ & $\begin{array}{l}\text { The coefficient for the radiated power necessary to } \\
\text { transmit in Free Space Channel (FSC) model. }\end{array}$ \\
\hline$\beta_{m p}$ & $\begin{array}{l}\text { The coefficient for the radiated power necessary to } \\
\text { transmit in Multipath Channel (MPC) model. }\end{array}$ \\
\hline$\rho$ & $\begin{array}{l}\text { The power consumption coefficient for receiving } \\
\text { data. }\end{array}$ \\
\hline$n_{s}$ & $\begin{array}{l}\text { The number of sensors connected to the cluster- } \\
\text { head. }\end{array}$ \\
\hline
\end{tabular}

In a nutshell, the complete heuristic search procedure is given in Fig.3 to compute the optimum number of sensors deployed $N_{S}$, the number of clusterheads deployed $N_{H}$, and the number of battery cells used in each type of device (i.e., $K$ and $k$ ) that minimizes the cost. While performing this search, we assume that the cost of the hardware component of clusterheads $C_{H W}$ is identical to that of sensor nodes. Thus, the main difference between these devices is that clusterheads are more energetic than sensor nodes. This energy differentiation is assumed to have discrete values. The rationale behind this consideration is that devices in WSNs are usually equipped with the "off the shelf" type of batteries which consist of a discrete number of battery cells. In Fig.3, $M A X_{C H}$ can be found by setting $N_{S}=0$ in Eqn.1 to Eqn.4. $M I N_{C H}$ can be found by setting $N_{S}=\infty$; this will lead to $S_{\text {cluster }}=\pi\left(r_{t s}+r_{s}\right)^{2}$, and the solution for $N_{H}$ was found accordingly.

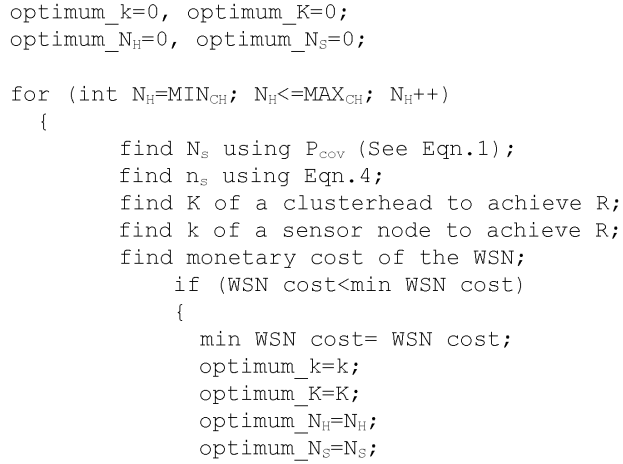

Fig. 3. Algorithm for Heuristic Search

\section{NUMERICAL RESUltS AND VALIDATION}

As a sample case, we used the values given in Table.II to solve the optimization problem. In this sample case, the number of rounds, $R$, is chosen such that sensor nodes die after approximately $R$ rounds (independent of the clusterheads' lifetime). That is, every sensor node has one battery cell which is sufficient for $R$ rounds.

The solution gives $N_{H}=3, N_{S}=23, K=79$, and $k=1$. In other words, a WSN consisting of 3 clusterheads and 23 sensor nodes all having a sensing range of $20 \mathrm{~m}$ will cover a sensing field with the dimensions $100 \mathrm{~m} \times 100 \mathrm{~m}$ with the probability of at least 0.9 . Although clusterheads have hardware identical with that of sensor nodes, their battery should contain 79 times more cells than the sensors' batteries to satisfy the targeted lifetime requirement. The cost of the WSN vs $N_{H}, N_{S}$ pairs satisfying coverage requirement is depicted in Fig.4. From this figure, it is also seen that $N_{H}=3$, $N_{S}=23$ pair leads to the optimum cost.

We also performed a computer simulation to validate our formulation and the solution. In the simulations, we used the solutions obtained from the heuristic search and found out the lifetime of the sensor network (i.e., the number of rounds). In the simulations, the number of experiments for each achieved 
TABLE II

SAMPle VAlues FOR HeUristic SEARCH

\begin{tabular}{|l|l|l|l|}
\hline Parameter & Value & Parameter & Value \\
\hline Sink $(X, Y)$ & $(50,175)$ & $P_{\text {cov }}$ & 0.9 \\
\hline$R$ & 12000 rounds & $r_{s}$ & $20 \mathrm{~m}$ \\
\hline$r_{t s}$ & $60 \mathrm{~m}$ & $r_{\text {th }}$ & $182 \mathrm{~m}$ \\
\hline$D$ & $100 \mathrm{~m} \times 100 \mathrm{~m}$ & $e_{\text {cell }}$ & $2 \mathrm{j}$ \\
\hline$C_{H W}$ & $\$ 3$ & $C_{\text {cell }}$ & $\$ 0.3$ \\
\hline$\rho$ & $50 \mathrm{Nj} / \mathrm{b}$ & $\alpha$ & $50 \mathrm{Nj} / \mathrm{b}$ \\
\hline$\beta_{f s}$ & $10 \mathrm{pJ} / \mathrm{b} / \mathrm{m}^{2}$ & $\beta_{m p}$ & $0.0013 \mathrm{pJ} / \mathrm{b} / \mathrm{m}^{4}$ \\
\hline$R_{S Y N C}$ & 50 byte & $R_{A G G}$ & 1000 byte \\
\hline$R_{J O I N}$ & 50 byte & $R_{S C H E}$ & 100 byte \\
\hline$R_{A D V}$ & 50 byte & $R_{D A T A}$ & 500 byte \\
\hline$E_{A G G}$ & $5 \mathrm{Nj} / \mathrm{b} /$ signal & & \\
\hline
\end{tabular}

round value is determined according to a confidence interval of $\pm 5 \%$ with the probability of 0.95 . We found that the average number of achieved rounds is 11372, whereas the targeted lifetime in the heuristic search method was 12000 . This result reveals that there is at most $5.23 \%$ discrepancy between targeted lifetime and simulated lifetime when optimum values are used. We believe that this discrepancy is acceptable because our cost optimization formulation assumes that each clusterhead is connected to a fixed number of sensors. However, in reality, due to the random deployment of devices, it cannot be guaranteed that every clusterhead has the same number of cluster members. Thus, in the simulation, some clusterheads die earlier.

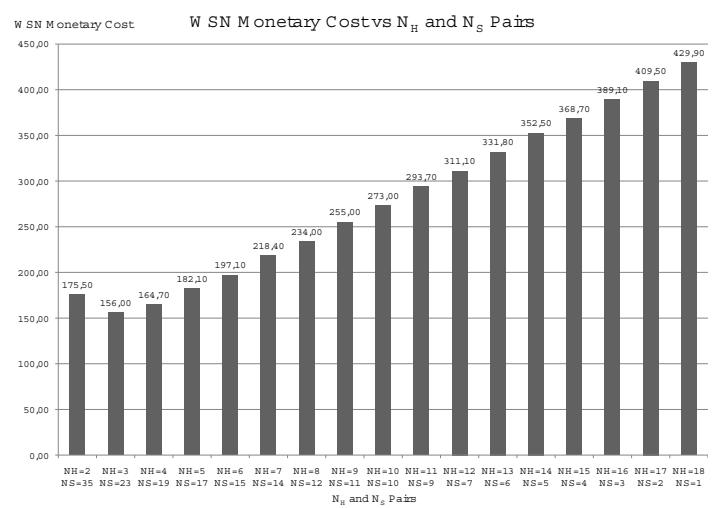

Fig. 4. Optimum WSN Cost

We also performed extensive simulations to validate our heuristic solution for different coverage requirements. Fig.5 shows the simulated and targeted lifetime values for various partial coverage values. For the lifetime validation, when the targeted lifetime value is 12000 , the $0.75,0.8,0.85,0.9,0.95$, and 0.99 partial coverage values exhibited $2.93 \%, 3.77 \%$, $5.07 \%, 5.23 \%, 6.73 \%$, and $10.73 \%$ errors, respectively. The results indicate that the error in the number of rounds increases as the coverage probability and/or the targeted lifetime increases. This is mainly due to the increase in the number of sensor devices in each cluster to satisfy better coverage. Therefore, our solution performs better under partial coverage with relatively small coverage probability requirement.

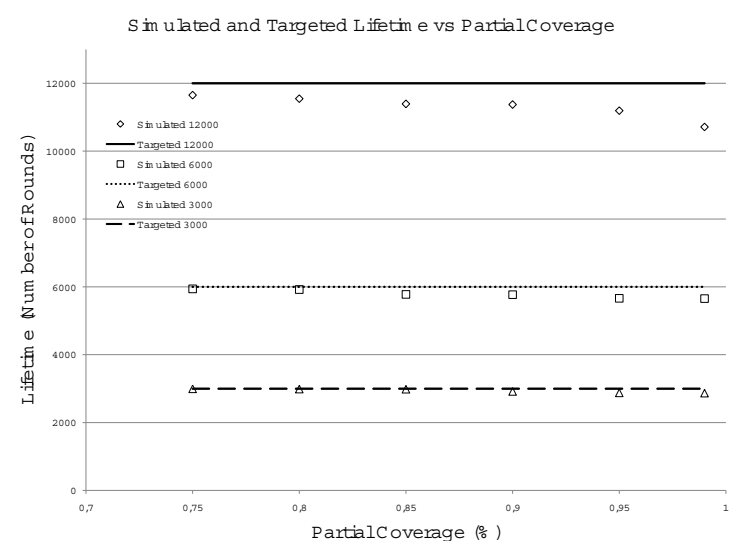

Fig. 5. Simulated and Targeted Lifetime

\section{CONCLUSION}

In WSN applications, it is usually common to have scarce resources, and the proper dimensioning of resources is extremely critical. Therefore, there is a need to look from the perspective of optimization as a whole, at a number of issues that have an impact on the WSN's ability to live long, that have cost within the anticipated budget, and that satisfy the coverage and connectivity requirements. In this wider context, we provide a generic framework to optimize these resources in randomly deployed WSNs. We believe that our optimization formulation can be used to aid researchers and practitioners to estimate the total cost of WSN for a given targeted lifetime, required minimum coverage, and other performance parameters

In this paper, we assumed that in clusterheads and in sensor nodes, we used identical but different numbers of cells in each type of device. Therefore, a large number of cells needs to be installed in clusterheads. Instead of using identical cells, we can use higher capacity cells in clusterheads and the required number of such cells in the clusterheads can be reduced. Usually there is a non-linear relationship between the capacity of the cell and its price, hence the cost of such a configuration would be lower too. Issues related to this relation are currently under study.

\section{REFERENCES}

[1] X. Liu, "Coverage with Connectivity in Wireless Sensor Networks", $3^{\text {rd }}$ International Conference on Broadband Communications, Networks and Systems, (BROADNETS 2006), Vol.1, pp. 1-8, 2006.

[2] W. Heinzelman, A. Chandrakasan, and H. Balakrishnan, "An ApplicationSpecific Protocol Architecture for Wireless Microsensor Networks", IEEE Transactions on Wireless Communications, Vol.1, No.4, pp. 660-670, October 2002.

[3] C. Sevgi, A. Koçyig̃it "On Determining Cluster Size of Randomly Deployed Heterogeneous WSNs" IEEE Communications Letters, Vol.12, No.4, pp. 232-234, April 2008.

[4] M. Gun, R. Kosar, and C. Ersoy, "Lifetime optimization using variable battery capacities and nonuniform density deployment in wireless sensor networks" $22^{\text {nd }}$ International Symposium on Computer and Information Sciences (ISCIS 2007), pp. 1-6, 2007. 